\title{
El diseño de recursos didácticos digitales: criterios teóricos para su elaboración e implementación
}

\section{The design of digital teaching resources: theoretical criteria for their development and implementation}

DOI: https://doi.org/10.32870/dse.v0i22.918

Verónica Pérez-Serrano Flores*

\begin{abstract}
Resumen
El presente ensayo tiene el propósito de desarrollar un diálogo argumentativo en torno a criterios teóricos que se pueden considerar para el diseño e implementación de recursos didácticos digitales. La importancia de reflexionar sobre el tema radica en la necesidad de mejorar la instrucción en línea, que por causa de la pandemia provocada por el virus del SARS-CoV2, ha impuesto la necesidad de trasladar la instrucción presencial a otras modalidades que se incluyen en el e-learning, tales como la educación línea o la educación mixta. La modalidad e-learning impone nuevos retos para el diseño no solo de la instrucción per se, sino de los recursos didácticos que acompañan dicha instrucción. Así, todos los actores educativos están en la búsqueda de adaptarse al e-learning: unos buscan traspasar su actuación educativa a la modalidad en línea sin realmente cambiar o reflexionar en cómo sus actividades didácticas no pueden replicarse en un formato digital; otros centran su actuación en reflexionar precisamente lo contrario; en el qué y en el cómo cambian sus actuaciones didácticas una vez que estas son mediadas por recursos digitales, principalmente Internet. Se considera que con la expansión de alternativas didácticas surgidas por el cambio de la modalidad educativa presencial a la modalidad e-learning, es necesario explorar algunos temas teóricos que podrían ser considerados como marco de referencia previo para apoyar la elaboración e implementación de recursos digitales principalmente en la educación básica.
\end{abstract}

Palabras clave: Recursos didácticos digitales - criterios teóricos.

\section{Abstract}

The purpose of this essay is to develop an argumentative dialogue around theoretical criteria that can be considered for the design and implementation of digital teaching resources. The importance of reflecting on the subject lies in the need to improve online instruction, which, because of the pandemic caused by the SARS-CoV2 virus, has imposed the need to transfer face-to-face instruction to other modalities included

\footnotetext{
* Doctora en Educación. Líneas de Investigación: Medios digitales y aprendizaje. Profesora de asignatura, Escuela de Pedagogía, Universidad Panamericana. Instituto Superior de Investigación y Docencia para el Magisterio, Secretaría de Educación del Estado de Jalisco. México. vperezs@up.edu.mx
} 
in the e-learning, such as online or blended education. The e-learning imposes new challenges for the design not only for the instruction per se, but of the teaching resources that accompany such instruction. Thus, all educational actors are in the search to adapt to e-learning: some seek to transfer their educational performance to the online modality without really changing or reflecting on how their teaching activities cannot be replicated in a digital format; others focus their performance on reflecting precisely the opposite; on what and how their teaching performances change once they are mediated by digital resources, mainly the Internet. It is considered that with the expansion of didactic alternatives arising from the change from face-to-face educational modality to the e-learning modality, it is necessary to explore some theoretical topics that could be considered as a prior reference framework to support the development and implementation of digital resources mainly in basic education.

Keywords: Digital teaching resources - theoretical criteria.

\section{La brecha digital}

Se hace evidente que el primer criterio para el diseño e implementación de recursos didácticos digitales es considerar las posibilidades de acceso a la infraestructura necesaria, esto es al hardware y al software que materializan la elaboración y el uso de un recurso digital. Por ello es imperativo revisar el concepto de brecha digital.

Este concepto es comúnmente definido como "la distancia que existe entre las personas que tienen y que no tienen acceso a las tecnologías de la información y comunicación" (Van Dijk, 2017: 1). En sus inicios, el concepto aplicaba solo al estudio sobre el acceso físico: obtener el hardware y el software de los medios digitales y la conexión a Internet. El acceso físico se correlaciona con indicadores demográficos tales como ingreso, nivel de educación, edad y género, cuestiones ampliamente discutidas como capital social en el campo de la sociología.

Posteriormente, en el año 2015, aparece en la escena del campo de la comunicación y los medios, un segundo nivel de brecha digital que se refiere a la brecha en el uso de los recursos digitales. Van Dijk (2017), en su teoría de recursos y apropiación expone la problemática de las brechas digitales y la sintetiza en los siguientes enunciados:

1. Las desigualdades sociales producen una inequitativa distribución de los recursos.

2. Una distribución inequitativa de los recursos causa un acceso desigual a las tecnologías digitales.

3. Un acceso desigual en las tecnologías digitales depende también de las características de dichas tecnologías.

4. La desigualdad en el acceso a las tecnologías digitales conlleva una participación también desigual en la sociedad.

5. Una participación desigual en la sociedad refuerza las desigualdades en la distribución de recursos. 
Quizás esté por demás señalar que, debido a la diversidad social y económica, la brecha digital de acceso está presente. Situación que se agrava aún más en la emergencia sanitaria que se está viviendo. Por lo que no se puede dar por sentado que los destinatarios de la instrucción (familias y alumnos) que componen el sistema educativo jalisciense del nivel educativo básico tienen acceso a las tecnologías digitales necesarias para recibir la instrucción en una modalidad virtual.

Así, podría resultar conveniente conocer censalmente quiénes (tanto discentes como docentes) tienen de facto acceso a las tecnologías digitales, ya que suponer que los principales agentes educativos poseen los medios económicos para recibir instrucción virtual sería una omisión que causa incertidumbre y agobio entre los padres de familia; muchos de los cuales, ya sin fuente de ingresos debido a la pandemia, no pueden asegurar el acceso a Internet debido a sus costos.

Si bien es cierto que estadísticamente la mayoría de la población tiene al menos una pantalla de teléfono inteligente, la conexión por este medio (por ejemplo, comprar acceso online o datos celulares por medio de una compañía telefónica) es bastante onerosa y poco práctica para trabajar contenidos educativos.

De la misma forma, habría que considerar que, aunque los docentes de enseñanza básica pertenecientes a la educación pública tienen su salario todavía asignado, la inversión personal que tienen que realizar para tener acceso a las tecnologías digitales, principalmente una conexión a Internet y con suficiente ancho de banda para atender al alumnado, no deja de ser un esfuerzo extra que habría que reconocer socialmente.

Se considera que las circunstancias mencionadas se podrían tener en mente en el diseño de recursos digitales educativos. Evidentemente, no hay solución inmediata a este problema; sin embargo, tener una estrategia para asegurar el acceso a los recursos educativos virtuales sería deseable ya que hasta ahora, por la premura de trasladar la instrucción presencial a la virtual, se están diseñando recursos digitales bajo el supuesto de que los agentes educativos tienen asegurado el acceso a dichos recursos. ${ }^{1}$

Por otro lado, para los educandos que sí tienen acceso a Internet, es imperativo considerar qué tipo de dispositivo digital están utilizando ya que el affordance ${ }^{2}$ de cada uno de ellos implica pensar en que los recursos educativos pudieran ser diseñados para operarse en múltiples pantallas.

Asimismo, es pertinente considerar que el acceso a las competencias digitales, llamadas también habilidades de alfabetización digital o mediática, es bastante desigual, simplemente

1 Para sustentar esta aseveración basta explorar los recursos didácticos que se encuentran disponibles en la página web de la Secretaría de Educación del Estado de Jalisco, llamada Recrea Digital (Cfr. https://recreadigital.jalisco.gob.mx/\#herramientas) y contrastarlo con el análisis de la marginalidad digital en nuestro estado donde se asienta que:"en Jalisco, [...] las diferencias de acceso y uso de la TIC están presentes, o al menos lo estaban muy claramente tomando como referencia los años 2010-2015. [...] La marginalidad está presente en todos y cada uno de los municipios estudiados" (Arredondo Ramírez, 2017: 162-163).

2 Capacidad de un objeto o ambiente para realizar una acción.

Diálopos sobre Educación 
por la diferencia generacional entre alumnos y docentes. Aquí cabe hacer la distinción entre habilidades instrumentales u operativas y habilidades relacionadas con el contenido.

El poseer las habilidades instrumentales (Ilamadas habilidades relacionadas con el medio) es decir, ser capaz de operar un medio y navegar por Internet, parecería que es condición suficiente para asegurar el acceso y, según la concepción popular, este tipo de habilidades son las que predominan entre la población. No obstante:

\begin{abstract}
Todas las investigaciones contemporáneas sobre la alfabetización o habilidades digitales han encontrado que habilidades como el recuperar información, la comunicación y la creación de contenido (llamadas habilidades relacionadas con el contenido) son de hecho más importantes que el mero uso de los medios. Tener habilidades relacionadas con el medio es solamente una condición previa para aplicar las habilidades relacionadas con el contenido (Van Dijk, 2017: 7).
\end{abstract}

Para reforzar esta noción, autores como Martin (2008) hablaban de niveles de alfabetización digital, los cuales se representan en la figura 1.

Figura 1. Niveles de alfabetización digital

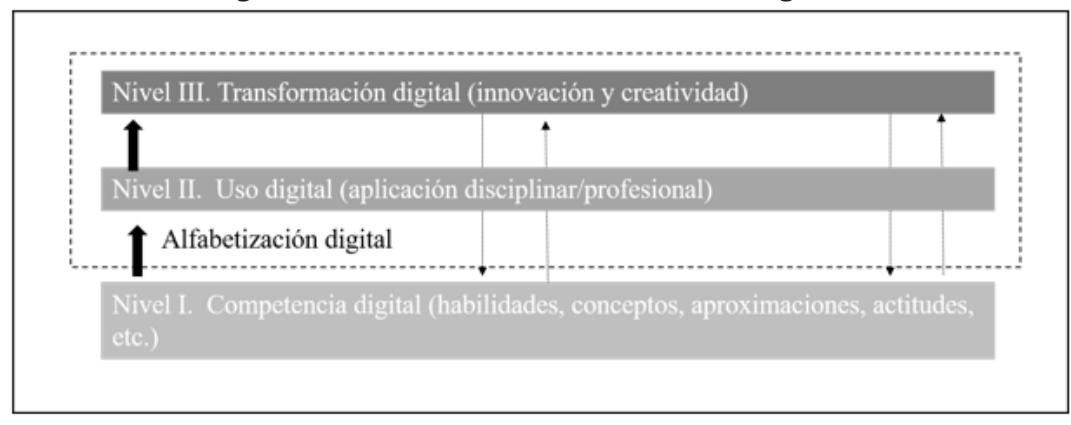

Fuente: Martin, 2008: 167.

Como se puede observar, no es lo mismo el ser alfabetizado digital que tener una competencia digital. Por lo tanto, para que el diseño de recursos didácticos digitales pueda ser oportuno hay que considerar que en realidad operar una tecnología digital y hacer uso de ella para enseñar y aprender son dos realidades diferentes.

En cuanto a los alumnos, la retórica alrededor de los "nativos digitales" (Prensky, 2001) hace creer que son eficientes en el manejo de las tecnologías digitales, lo cual puede ser cierto si se hace referencia al nivel I de la alfabetización digital o a las habilidades instrumentales u operativas. Pero esto no aplica necesariamente a las habilidades propias de un individuo que es verdaderamente alfabetizado digital o que posee las habilidades relacionadas con el contenido.

La contra evidencia de la existencia de esta retórica, que indica la capacidad casi innata de los estudiantes para manejar una tecnología digital, es clara: "mientras algunos niños o jó-

Diálo@os 
venes pueden sentirse muy confiados en el uso de la tecnología, su entendimiento de cómo la tecnología funciona y cómo puede ayudarles a aprender puede ser extremadamente limitada" (Coombes, 2009; Kennedy et al., 2009; Singh, Mallan, Giardina, 2008, como se citó en Bennet, Maton, 2011, sección "Research about Digital Natives").

Lo mismo sucede con los docentes. Es posible que muchos tengan ya desarrolladas las habilidades instrumentales u operativas, pero no las habilidades relacionadas con el uso de los contenidos mediáticos.

Ciertamente la gran mayoría de los docentes está logrando pasar en un tiempo récord del nivel 1 de competencia digital al nivel 2 y 3 de alfabetización digital, es decir, a un uso disciplinar y creativo de los recursos digitales. Como ejemplo están todos los recursos digitales compartidos en las redes sociales de los docentes.

Para concluir este apartado, solo se podría agregar que los problemas que imponen el acceso y uso de recursos digitales (brecha digital) no son unos que se vayan a resolver ni siquiera a mediado plazo. Sin embargo, las condiciones actuales impuestas al sistema educativo obligan a pensar en estrategias a corto plazo, que se presentan a continuación a manera de ideas propositivas:

1) En el diseño de recursos digitales se tendría que considerar una estrategia alterna para disminuir la desigualdad en la brecha de acceso, es decir, ver la posibilidad de utilizar otros medios asequibles.

2) En caso de no existir brecha en el acceso y tener recursos digitales disponibles, diseñarlos en múltiples formatos para que puedan ser leídos y utilizados en múltiples pantallas, considerando el affordance de cada medio digital ( $p c$, tabletas digitales o teléfonos inteligentes).

3) Para lograr la implementación de recursos no solo se requiere diseñalos, sino también llevar a la par la formación digital de los agentes educativos (padres, docentes y alumnos) para que logren pasar el uso instrumental de los recursos (por ejemplo, localizar un recurso en un repositorio) a la creación de contenidos. Esta situación emergente de la pandemia abre la posibilidad de romper con la resistencia de los agentes educativos a emplear y crear recursos, siempre y cuando exista un acompañamiento y un adecuado andamiaje que no tenga como único objetivo la entrega digital de recursos, sino el hacer uso de la llamada "inteligencia colectiva" y la cooperación entre las "multitudes inteligentes" (Rheingold, 2004) para crear verdaderas comunidades de práctica.

4) Relacionado con el punto anterior, la creación existosa de comunidades que son capaces de organizar y controlar su conducta, en este caso la manera en que se están compartiendo los recursos digitales comunes, tienen características distintivas útiles en la formación digital y el diseño de recursos didácticos. La socióloga Elinor Ostrom 
defendió que no siempre son necesarias las autoridades externas para el control de los recursos, lo que ella denomina recursos comunales (common pool resources). Ostrom (1990) compararó distintas comunidades y aquellos grupos que resultaron más existosos se rigen por los siguientes principios:

- Se definen claramente los límites del grupo.

- Las normas que rigen el uso de los bienes colectivos responeden bien a las necesidades y condiciones del lugar.

- La mayoría de los individudos sujetos a estas normas pueden participar en la modificación de las mismas.

- Las autoridades externas respetan el derecho de los miembros de la comunidad a definir sus propias normas.

- Existe un sistema para controlar la conducta de los miembros; los propios miembros de la comunidad ejercen ese control.

- Se emplea un sistema de sanciones graduado.

- Los miembros de la comunidad tienen acceso a mecanismos poco costosos de resolución de conflictos (Hess, Ostrom, 2007: 7).

Así, existe la posibilidad de confiar en los docentes de educación básica para que logren conformar comunidades donde los recursos didácticos sean elaborados y compartidos, pero siempre con manifiesto respeto a la autonomía y organización de los grupos de aprendizaje que los docentes están construyendo para apoyar su labor educativa en estos tiempos de crisis.

\section{Contenido educativo}

Respecto al contenido educativo, se entiende la necesidad de darle continuidad a los campos de formación académica o área personal y social curriculares, asentados en los programas del nivel educativo básico. Sin embargo, ya que la entrega de estos contenidos tiene ahora una lógica que involucra forzosamente el uso de las tecnologías digitales, el contenido educativo y la forma de producirlo y trasmitirlo se trasforma necesariamente.

El modelo de conocimiento tecnológico, pedagógico y de contenido conocido como TPACK (Technological Pedagogical Content Knowledge, por sus siglas en inglés), (Koehler, Mishra, 2009), aunque pudiera parecer temporalmente antiguo, clarificó cómo el contenido y la pedagogía se trasforman cuando entran en interacción con la tecnología. ${ }^{3}$

Para ello, el TPACK considera, primero, que enseñar con tecnología representa para los docentes retos importantes debido a que las propiedades inherentes de los recursos digitales hacen que su aplicación estandarizada sea difícil. A continuación se describen dichas propiedades:

3 Al utilizar el modelo TPACK se logra el diseño de una actividad didáctica que, mediada tecnológicamente, puede ser más efectiva para promover el aprendizaje. 
Las tecnologías pedagógicas tradicionales, a diferencia de las tecnologías digitales, están caracterizadas por su especificidad (un lápiz se utiliza para escribir); estabilidad (el uso del pizarrón no ha cambiado prácticamente desde su invención) y transparencia en su función (la forma en como funciona un lápiz está directamente relacionada con su función). Mientras que las tecnologías digitales son proteicas (utilizadas en diferentes formas), inestables (cambian rápidamente) y opacas (su funcionamiento es oculto a los usuarios) (Koehler, Mishra, 2009: 61).

De las características de las tecnologías digitales mencionadas, quizá las que más alteren a los docentes de educación básica son sus cualidades proteicas e inestables. Si las tecnologías digitales pueden ser utilizadas en miríadas de formas, y además están en constante cambio, la estrategia de producir contenidos digitales podría considerar que dichos contenidos serán utilizados también de forma diferente y que en definitiva tendrán fecha de caducidad.

Por ejemplo, las presentaciones estáticas de contenido están pasando, gracias al uso de la web 2.0, a ser más interactivas. El software online, como Nearpod, o Mentimeter, permite que los alumnos interactúen con la presentación de manera más dinámica, a través de preguntas que han de responderse o de introducción de espacios de discusión sobre el propio contenido.

La presentación de contenido a través de videos o tutoriales también mira cada vez más a la interactividad con los estudiantes, al igual que a través de programas online como Playposit, que posibilitan la creación de preguntas, recortes del video, introducción de comentarios por parte de los estudiantes, etcétera.

Con la inclusión de la interactividad, la creación de contenidos educativos estáticos se utilizará de maneras diferentes y cambiará constantemente. Por ello, no se puede esperar que un contenido de esta naturaleza, incluido en un repositorio, sea utilizado de manera estandarizada, lo cual no implica que no se considere que el objetivo de aprendizaje sea cubierto con independencia del tipo de contenido.

Parece entonces necesario tomar en cuenta que cualquier contenido que se produzca pronostique que su uso será sumamente maleable, por lo que lo más importante es centrar la atención, no en el contenido curricular en sí, sino en el objetivo de aprendizaje que se desea alcanzar con él.

Otra consideración, mencionada al principio de este apartado, es que el contenido educativo y la pedagogía cambian cuando interactúan con la tecnología. Estos cambios son precisamente los que explica el modelo TPACK de Koehler y Mishra (2009), que a continuación se resumen:

1. El conocimiento del contenido (Content Knowledge o CK por sus siglas en inglés): nos habla del saber que tienen los docentes sobre la materia que están enseñando, es decir, hechos, teorías, conceptos, perspectivas históricas. 
2. El conocimiento pedagógico (Pedagogical Knowledge o PK): se refiere a saber cómo se está enseñando o cómo se va a enseñar. Esto incluye conocimientos de procesos, prácticas y métodos de enseñanza-aprendizaje, propósitos educativos y valores, e implica también saber cómo el estudiante aprende, las técnicas y métodos por utilizar y las teorías de aprendizaje.

3. El conocimiento tecnológico (Technological Knowledge o TK): es más que tener habilidades digitales ya que implica un dominio de la tecnología para procesar información, comunicarse y resolver problemas.

Ahora bien, estos tres conocimientos son básicos para crear actividades a través de medios digitales, pero no son suficientes. Cuando interactúan estos tres componentes se modifican produciendo nuevos tipos de conocimiento:

4. El conocimiento pedagógico y de contenido (Pedagogical Content Knowledge o PCK): se refiere a la trasformación que sufre la materia o el contenido cuando el maestro interpreta el contenido y encuentra múltiples maneras de representarlo. Es básicamente el centro de la enseñanza.

5. El conocimiento tecnológico y de contenido (Technological Content Knowledge o TCK): implica entender el impacto de las tecnologías en el desarrollo de las disciplinas que se enseñan, ya que lo que se sabe del contenido de una materia cambia, en parte, por el desarrollo de las tecnologías.

6. El conocimiento tecnológico y pedagógico (Technological Pedagogical Knowledge o TPK): es el conocimiento que permite entender cómo la enseñanza puede cambiar cuando una tecnología se utiliza de cierta manera. Este conocimiento es muy importante, sobre todo porque las tecnologías digitales no fueron creadas para propósitos educativos, sino que se han adoptado y adaptado.

Finalmente, solo si se consideran los tres tipos de conocimiento (de contenido, pedagógico y tecnológico) más sus interacciones (conocimiento pedagógico y de contenido, conocimiento tecnológico y de contenido y conocimiento tecnológico pedagógico) es posible crear una actividad TPACK, es decir, aquella actividad que se considera la base de la enseñanza efectiva con tecnología, ya que es la forma como se representan los contenidos curriculares a través de medios tecnológicos.

Así, lo más importante para este modelo no es la tecnología, sino el saber la materia a enseñar, a la vez que se emplea la pedagogía para construir conocimiento. De hecho, en una actividad TPACK lo primero que se tiene que saber es el contenido, posteriormente la pedagogía y solo entonces se puede incluir la tecnología. 
A continuación, se representa el modelo TPACK de Koehler y Mishra (2009) (figura 2).

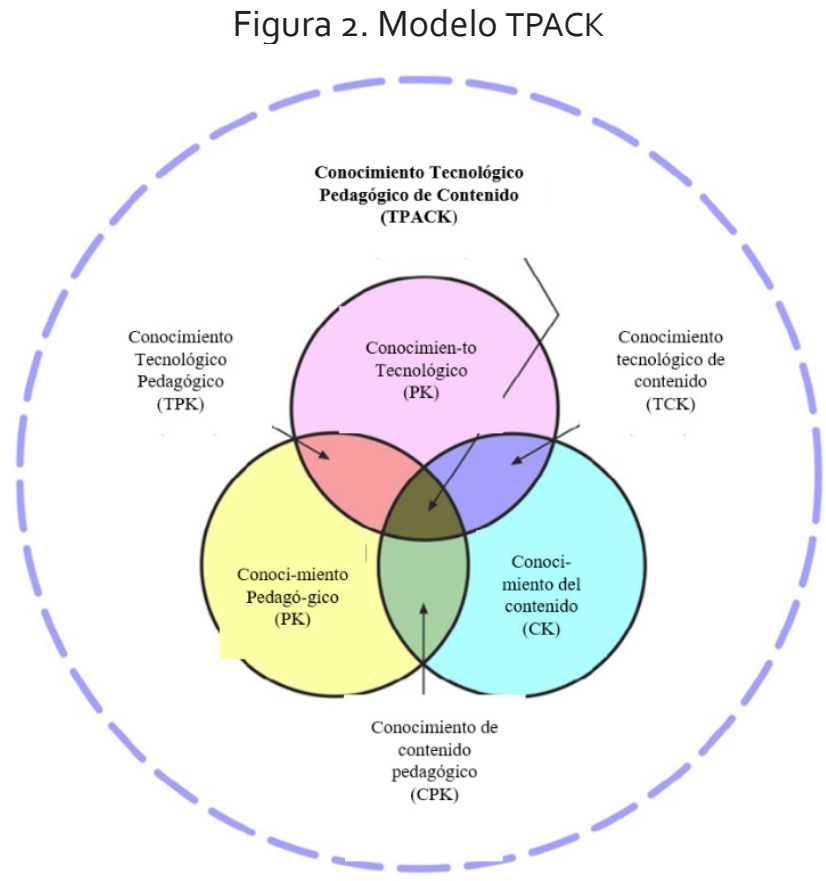

Fuente: Koehler y Mishra (2009).

Sirva este modelo para considerar que el traspaso de la instrucción cara a cara a la instrucción virtual no es trasparente, es decir, la creación de recursos digitales desprovistos de conocimiento curricular y de pedagogía serán poco útiles para la instrucción virtual.

Por ello, merece la pena pensar en que la implementación y la creación de recursos digitales implican siempre una movilidad de los modelos pedagógicos cuando son mediados por las tecnologías digitales.

Además, es un error suponer que hay que "vaciar" el conocimiento y la pedagogía en un recurso didáctico; más bien la lógica es la contraria: la formación digital empieza cuando los maestros reflexionan que primero está el saber qué se va a enseñar y el cómo se va a enseñar y luego -solo entonces- se puede pensar en la tecnología o en diseñar un recurso didáctico digital.

Así, parece conveniente sugerir que la implementación y diseño de recursos digitales empiece por establecer algún modelo que explique la lógica de construcción y entrega de la instrucción a los alumnos de cualquier lección mediada por tecnología.

Esto nos demuestra que cuando se trata de enseñar online, las tecnologías no funcionan simplemente como instrumentos que asisten al trabajo instruccional. En otras palabras, como 
indica Major (2015), las tecnologías no son el respaldo de la instrucción, sino el contexto de esta. Y como todo contexto o "lugar", el mundo virtual es el lugar donde ocurre el proceso de enseñanza-aprendizaje.

En una situación ideal, enseñar online podría ser una opción para muchos docentes que poseen competencias digitales suficientes; sin embargo, esta no es la condición actual. Pese a que las autoridades educativas mexicanas han impulsado diferentes reformas educativas que intentan responder a las exigencias sociales derivadas de la revolución tecnológica, la actualización docente en materia de alfabetización tecnológica sigue apareciendo en las reformas impulsadas por el estado (Rosales Medrano, 2009).

De esto se infiere que la adquisición suficiente de competencias tecnológicas es un proceso que ya se ha iniciado y necesita continuar, especialmente ahora que los docentes están comprometidos con un cambio de gran calado, que además se tiene que implementar sin los recursos adecuados, ni infraestructurales ni temporales. ${ }^{4}$

Según Major (2015), algunas preguntas que podrían considerarse para la formación digital de los agentes educativos cubren las siguientes áreas:

a) Evaluar la tecnología con la que se cuenta: enseñar online requiere de una adecuada tecnología y para evaluar este aspecto se debería reflexionar en las siguientes preguntas:

- ¿Se tiene acceso a una computadora confiable?

- ¿Acceso a Internet de alta velocidad?

- ¿Acceso a sitios o herramientas que soporten la instrucción?

b) Evaluar la estructura de soporte de la institución:

- ¿Hay oportunidades de entrenamiento por parte de la institución?

- ¿Los maestros tienen el soporte de sus colegas para responder a las preguntas acerca de la tecnología o pedagogía que se requiere para enseñar online?

Estas dos últimas preguntas sirven de respaldo para seguir implementando cursos para diseñar y emplear recursos digitales. Evidentemente, no hay tiempo de pausar el curso de la situación y dar entrenamiento a todos los agentes educativos, pero mínimamente se podría considerar que, a la par de la exigencia actual por evidencias del trabajo en ambientes digitales, los agentes sientan también que hay un respaldo en cuestiones de capacitación o entrenamiento.

Una sugerencia es abrir la posibilidad de organizar cursos masivos online o abiertos (Massive Online Open Courses o MOOC's por sus siglas en inglés) o utilizar algunos ya elaborados y que tengan como aliciente no solo el adquirir habilidades, sino también que puedan tener algún valor curricular para los docentes.

4 Se refiere a la brecha digital tanto en la posesión y uso de recursos como a la implementación acelerada de educación en línea debido a la contingencia sanitaria por el virus SARS-CoV2.

Diálopos sobre Educación año 12 | número 22 | enero-junio 2021 | ISSN 2007-2171 
c) Evaluar las habilidades:

- ¿Tienen los agentes educativos buenas habilidades de organización?

- ¿Tienen los agentes educativos habilidades para organizar su tiempo?

- ¿Tienen los docentes habilidades para dar retroalimentación rápida a sus estudiantes?

- ¿Tienen los agentes educativos habilidades para plasmar sus pensamientos en forma escrita?

- ¿Existen evidencias de que se manejan y entienden los términos relacionados con la tecnología computacional?

- ¿Tienen los agentes educativos las habilidades técnicas necesarias para manejarse en un ambiente virtual? (por ejemplo, escribir en un teclado, manejo de sistema de archivos -guardar, crear, organizar archivos-, manejo de procesador de textos, manejo de software de presentaciones o manejo de redes sociales).

Sería importante conocer la valoración actualizada de estas habilidades en los agentes educativos a fin de tener un punto de partida para conformar los criterios de diseño para la implementación y elaboración de recursos didácticos.

Como se ha argumentado en este apartado, parece necesario que los criterios de contenido educativo, además de los mencionados en el documento, puedan considerar: a) un modelo de trabajo para implementar el trabajo con tecnologías digitales y b) hacer una evaluación de los recursos necesarios para operar la instrucción online.

\section{Entender los medios o recursos para la enseñanza}

Además de las reflexiones anteriores, puede ser útil establecer una conceptualización sobre los medios en la toma de decisiones del diseño. Según Romero Tena y Cabero Almenara (2007), hay tres perspectivas que marcan la evolución de los medios para la enseñanza:

1) Técnica.

2) Sistema simbólico.

3) Sistema de variables.

La perspectiva técnica se centra meramente en el carácter informativo y comunicativo del medio y se caracteriza por considerase un soporte material que trasmite información, por lo que su diseño irá siempre encamindo a representar información y persiguirá su trasmisión con una finalidad educativa.

La perspectiva que ve al medio como un sistema simbólico se centra tanto en los sistemas simbólicos que utiliza para codificar el mensaje como en sus posibilidades estéticas y técnicas, por lo que su función, en el mejor de los casos, es estructurar la información de tal forma que pueda adaptarse a las operaciones intelectuales que el sujeto receptor vaya a realizar. Así, 
en el diseño de medios se procura estructurar la información en procesos similares a los del pensamiento.

Finalmente, la tercera perspectiva, la del sistema de variables, pone el acento en la influencia que ejerce el ambiente y cómo este determina la integración del medio y las posibilidades de interacción con él. Entonces en su diseño se consideran las características del contexto donde va a ser utilizado porque influirá significativamente en los resultados que se logren con el medio diseñado.

A continuación se transcriben, de Romero Tena y Cabero Almenara (2007:33), los principios generales que pueden ser útiles para ampliar los criterios para el diseño:

1. Los medios son solamente unos elementos curriculares, que funcionan en interacción con otros y, en consecuencia, su significación en el proceso de enseñanza-aprendizaje dependerá de las decisiones que se adopten respecto al resto de componentes, al mismo tiempo que las decisiones tomadas sobre estos repercutirán en el resto de los componentes del sistema.

2. El contexto instruccional, físico, cultural y curricular son elementos que facilitan o dificultan, no sólo cómo el medio puede ser utilizado, sino también si debe serlo.

3. Su utilización requiere un proyecto pedagógico previo que le dé sentido y cobertura teórica.

Por lo tanto, en consonancia con lo expuesto sobre el diseño de actividades mediadas por tecnología (por ejemplo, el TPACK), los criterios, implementación y diseño de recursos didácticos podrían considerar primero y ante todo a la pedagogía, antes que a la propia tecnología. Así es útil explicitar no solo los propósitos del medio y las instrucciones para utilizarlo, sino también ciertas bases pedagógicas más concretas de cómo utilizarlo para que se conviertan en verdaderas herramientas de enseñanza y aprendizaje.

Evidentemente, esto implica que los sujetos que utilizarán un medio digital conozcan no solo cómo se manipula, sino también su base pedagógica común o instruccional. Si esta consideración sobre la pedagogía ya es difícil para profesionales (docentes y directivos) lo será aún más para los alumnos y los padres, por lo tanto, tendría que ser muy cuidada y explícita en el diseño de la formación digital de estos agentes.

En cuanto a la base pedagógica común, en el diseño sería deseable incluir también el tipo de actividades y el tiempo en que el estudiante estará interactuando con ellas.

Conole (2013) clasifica las actividades incluidas en la instrucción digital de la siguiente forma:

1) Actividades asimilativas (leer, escuchar, ver, por ejemplo leer información en una página web, escuchar un podcast, o ver un video en Youtube).

2) Actividades de manipulación de información (como por ejemplo utilizar un software de análisis estadístico tal como SPSS o Excel).

Diálo Dos

sobre Educación

TEMAS ACTUALES EN INUESTIGACLON EU año 12 | número 22 | enero-junio 2021 | ISSN 2007-2171 
3) Actividades de comunicación y productivas (crear un artefacto, por ejemplo, una presentación multimedia).

4) Actividades experienciales (practicar o imitar una habilidad que se puede lograr a través de un videojuego del género de estrategia) $y$,

5) Actividades adaptativas (modelar o simular, por ejemplo, utilizar un simulador de realidad virtual).

Cada una de estas actividades tiene en su base la utilización de diferentes habilidades de pensamiento.

Desde la publicación de la taxomía de Bloom y su posterior revisión realizada por Anderson y Krathwohl (2001: 63), se reconoce que: "dos de las metas educativas más importantes es promover la retención y promover la transferencia (que cuando ocurre, indica un aprendizaje significativo)".

Para lograr esas metas se emplean diferentes procesos cognitivos; relacionado íntimamente con la retención está el proceso cognitivo de recordar, mientras que los relacionados con la trasferencia se refieren a entender, aplicar, analizar, evaluar y crear.

En cuanto al tipo de actividades mencionadas, es posible establecer que existe una relación entre su tipología y la actividad cognitiva. Por mencionar las actividades de asimilación (leer, escuchar y ver), pueden promover diferentes procesos cognitivos, no sólo el recuerdo sino también entender, analizar y evaluar, siempre y cuando el objetivo instruccional del recurso didáctico digital vaya dirigido a promover no solo retención, sino también la trasferencia. Por ello sería conveniente agregar en los criterios de diseño el tipo de proceso cognitivo que se quiere promover.

Evidentemente, actividades de manipulación, comunicación y productivas, experienciales o adaptativas pudieran promover aún más la trasferencia, desde que involucran habilidades de pensamiento de orden superior (High Order Thinking Process o HOTS por sus siglas en inglés); a diferencia del mero recuerdo, que implica el despliegue de habilidades de pensamiento de orden inferior (Lower Order Thinking Process o LOTS por sus siglas en inglés) (Churches, 2008).

Por ello es que se mencionaba que considerar la conceptualización de recursos didácticos desde su perspectiva simbólica es muy importante ya que de lo que se trata es de empatar el diseño del recurso didáctico con el pensamiento del estudiante. Esta es solo una apreciación que se sugiere incluir en los criterios de diseño, así como la selección de una taxonomía adecuada de objetivos para la era digital ${ }^{5}$ que sea congruente con la perspectiva teórica curricular que se desee implementar.

5 El documento de Churches (2008), Bloom's Taxonomy Blooms Digitally, ofrece ideas de los tipos de recursos digitales y los tipos de habilidades de pensamiento que promueven. No obstante, podrían encontrarse otras taxonomías más actualizadas. 


\section{Concepción y recursos del e-learning}

Como reflexión teórica final, se cree necesario entender el tipo de modalidad educativa que acompaña la implementación y diseño de recursos didácticos digitales.

En primer término, es prioritario posicionarse teóricamente sobre la forma de entender el e-learning, ya que parece existir una confusión conceptual entre este y la modalidad en línea, el aprendizaje móvil y la modalidad mixta, entre otras. El e-learning ha evolucionado a la par de la tecnología, por lo que se pueden distinguir tres etapas en su desarrollo:

1) Primera generación: se centra en la elaboración de materiales específicamente diseñados para la formación en línea; el modelo de material tiene que ser didáctico e incluir elementos de reflexión, síntesis, actividades, etcétera. Entre las características de los materiales de esta primera generación se encuentran los contenidos en formatos de papel, contenidos digitales reproduciendo libros, audioconferencias, videoconferencias y el software instruccional.

2) Segunda generación: El énfasis se coloca en la creación de campus virtuales, focalizándose en plataformas y gestores de e-learning. En esta etapa los modelos educativos no tienen un papel relevante o son dependientes de las plataformas desarrolladas. Entre los materiales utilizados en esta segunda generación se encuentran los entornos virtuales de aprendizaje (como Moodle, Google Classroom, Blackboard), el videostreaming, los materiales en línea, el acceso a los recursos de Internet y se inicia con la interactividad a través del uso del e-mail y de los foros.

3) Tercera generación: el sentido de la formación en red se orienta hacia el trabajo en equipo y al estudiante se le pide que sea capaz de gestionar y producir conocimiento de forma conjunta. Se basa en la participación posibilitada por la Web 2.0, a través de la participación y construcción colectiva del conocimiento. Los materiales utilizados en esta tercera generación son contenidos especializados en línea y también generados por los estudiantes; reflexión (e-portafolios, blogs), tecnologías interactivas (juegos, simulaciones, visualización en línea), comunidades de aprendizaje en línea y el aprendizaje móvil (mobile learning o m-learning) (Gros, 2011: 15-16).

Cada institución educativa ha alcanzado diferentes etapas en este desarrollo dependiendo, claro está, de la modalidad educativa que ofrecen (presencial, híbrida o blended o en línea). Sin embargo, una caracterización popular del e-learning es la siguiente: "El e-learning se toma comúnmente para indicar el uso de Internet y las computadoras para aprender" (Littlejohn, Pegler, 2007: 16).

Así, con esto en mente, es casi imposible no utilizar el affordance y las posibilidades que ofrecen las tecnologías digitales (Herrington, Reeves, Oliver, 2010), especialmente por su ubi- 
cuidad $^{6}$ en la mayoría de las actividades cotidianas. Para ejemplificar esta idea se aclara que, según la Encuesta Nacional sobre Disponibilidad y Uso de Tecnologías de la Información en los Hogares (ENDUTIH, 2019), en México hay 80.6 millones de usuarios de Internet y 86.5 usuarios de teléfonos celulares, lo que representa $70.01 \%$ de la población de 6 años o más; entre las actividades que realizan los usuarios se encuentran la diversión (91.5\%), la obtención de información (90.7\%) y la comunicación (90.6) (INEGI, 2020).

Lo anterior no es ajeno a las instituciones de educación básica, que han sabido integrar el uso de la tecnología digital para proveer sus servicios educativos. No obstante, habría que diferenciar el e-learning y la modalidad de enseñanza en línea. Quizá los aspectos que más diferencian a la modalidad en línea son:

1) La asincronicidad: se refiere a que tanto los estudiantes como el docente no coinciden en un tiempo determinado para tomar una clase.

2) La flexibilidad: el estudiante aprende a su ritmo y el diseño instruccional de las lecciones ha de estar pensado para posibilitar el aprendizaje anytime y anywhere.

Entonces, como se puede observar, hay características diferenciadoras entre el e-learning y la modalidad de aprendizaje virtual, siendo el primero ya utilizado en la modalidad presencial. Se hace especial mención en este punto porque, debido a la contingencia sanitaria, se piensa que la migración de la modalidad presencial a la modalidad virtual implica de alguna manera que las "clases" transcurran en la sincronía planeada para cada curso y que por esta razón, tanto los estudiantes como los maestros tienen que estar "conectados" a la hora de la clase. Evidentemente esto no es educación en la modalidad virtual. Puede ser e-learning pero no enseñanza virtual.

Se considera que durante la contingencia sanitaria las estrategias para la implementación y diseño de recursos digitales han de responder más a la modalidad e-learning que a la educación virtual, esto por dos razones. La primera, es que la mayoría de los programas de las instituciones educativas del nivel educativo básico (con excepción del nivel medio superior) están diseñados curricularmente para ser ofertados de manera presencial. ${ }^{7} Y$ la segunda, la migración de la educación presencial a la educación virtual no puede ser sino escalonada, lo que significa que su implementación requiere de una inversión considerable de recursos tales como:

- Infraestructura tecnológica (adquisión de hardware y software para la creación de aulas virtuales y materiales y recursos digitales).

6 Ubicuidad se entiende como la posibilidad de acceder y procesar información en cualquier momento y lugar (anytime, anywhere) (Burbules, 2012; Cope, Kalantzis, 2009).

7 En la Ley General de Educación, en el capítulo IV, del Proceso Educativo, se menciona la modalidad no escolarizada, sin embargo no se ofertan lineamientos claros sobre la misma. Cfr. Diario Oficial de la Federación (2019). En este documento legal se hace patente el dominio del Estado mexicano sobre los contenidos y operación curricular presencial de las escuelas de educación básica tanto públicas como privadas incorporadas a la Secretaría de Educación Pública (SEP). 
- Capacitación profesional de los docentes.

- Contratación de expertos en diseño instruccional (los docentes no son, ni tienen por qué ser, diseñadores instruccionales).

- Contratación de expertos en el manejo de plataformas educativas.

- Contratación de servicios de consultoría para implementar las plataformas.

Evidentemente muchas instituciones educativas, especialmente las de sostenimiento público, carecen del tiempo y los recursos para migrar a la educación virtual, pero sí hay posibilidades de implementar el e-learning tal como se ha definido.

Otras instituciones estarán mejor preparadas y equipadas para migrar a la modalidad en línea, sin embargo, un punto clave para ello será que los programas curriculares estén diseñados para esta modalidad y que los docentes que los implementarán estén plenamente capacitados. Por ello, se asienta que es posible pensar en que la mayoría de prácticas educativas actuales caen dentro del e-learning y no completamente dentro de la modalidad en línea.

\section{Consideraciones finales}

Hasta el momento se abordaron criterios teóricos que habría que tomar en cuenta previamente para diseñar e implementar recursos didácticos digitales.

Los temas expuestos pretendieron aportar un marco referencial para pensar cómo se puede mejorar la instrucción en línea a partir del uso de diferentes recursos didácticos digitales y, sin ser un marco que contemple todas las aristas de enseñar en la modalidad e-learning, consideró aspectos clave como el acceso y uso de recursos, la pedagogía que debe guiar su implementación, la modalidad educativa y el tipo de actividades que es necesario diferenciar para entender cómo lograr mejores resultados de aprendizaje.

En cuanto a la brecha digital, tanto de acceso como de uso, habrá que reconocer que su presencia, principalmente entre los agentes que cursan y operan el nivel de educación básica, no puede pasar desapercibida. Quienes no tienen acceso infraestructural no podrán utilizar el recurso, y quienes sí tienen acceso, pero carecen de las habilidades de alfabetización digital o presentan brecha en el uso, tendrán dificultades para convertir el recurso digital en un medio que promueva de forma más cabal el aprendizaje, dado que no es didáctico por sí mismo.

El componente didáctico de un recurso digital se puede lograr cuando se utiliza de manera sistemática cualquier modelo instruccional que apoye la labor de enseñanza. Por ello, el modelo TPACK muestra que un recurso que pretenda entregar un contenido de aprendizaje a un estudiante, forzosamente se modifica porque está atravesado por la mediación: por un lado, la mediación de las posibilidades tecnológicas del software y el hardware que trasforma los contenidos de aprendizaje; y por el otro, la ausencia de mediación directa de un profesor que cambia las formas de enseñar. 
Adicionalmente, no solo es necesario utilizar un modelo instruccional para la creación de recursos digitales, sino también reconocer que los medios por sí solos no promueven el aprendizaje formal y significativo necesario para lograr su aplicación en contextos de la vida cotidiana. Lo que le otorga valor didáctico al medio es la pedagogía que lo sustenta.

Así, también es necesario identificar el tipo de actividades que se pueden incluir en la instrucción en línea y tener claro cómo llegar a la meta de lograr la trasferencia de los contenidos de aprendizaje a través del uso de los recursos digitales.

Por otro lado, para dar sentido a la enseñanza es útil reconocer la modalidad de enseñanza que se está utilizando, ya que es más factible entender que si se utiliza una modalidad que pretende ser virtual, la planeación de las actividades de aprendizaje cobra una importancia central, porque implica que la asincronicidad y flexibilidad características de esta modalidad trasformen el rol del docente: de ser un trasmisor a ser un diseñador de experiencias de aprendizaje. En cambio, si la modalidad que se utilizará es híbrida o sincrónica, el uso de los recursos digitales permite que la presencialidad del docente y de los alumnos, aun siendo mediada por la tecnología (por ejemplo, a través de una videoconferencia), provoquen un intercambio más inmediato de ideas que pueden llevar a la construcción del conocimiento compartido por el intercambio comunicacional directo entre los docentes y sus alumnos y entre los propios alumnos.

Finalmente, habrá que admitir que la premura provocada por la contingencia sanitaria, con la que los agentes educativos de educación básica están diseñando e implementado recursos digitales que acompañan a la modalidad e-learning, dejará huella en la forma en que se está educando a los estudiantes.

Ahora se está aprendiendo a aceptar que los roles tanto de maestros como de alumnos, están cambiando. Los primeros han de aprender a descolocarse de su rol como trasmisores y poseedores del saber para pasar a ser auténticos mediadores y diseñadores de experiencias de aprendizaje; y los segundos, tienen que dejar de ser receptores pasivos para convertirse en estudiantes más autónomos y responsables.

Finalmente, estas trasformaciones parecen ser ideales que se persiguen tanto en la educación virtual como en la educación presencial, independientemente de la crisis sanitaria actual.

\section{Referencias}

Anderson, L.; D. Krathwohl (eds.). (2001). A Taxonomy for Learning, Teaching and Assesing. A Revision of Bloom 's Taxonomy of Educational Objetives. Nueva York: Longman.

Arredondo-Ramírez, P. (2017). Conectividad y desigualdad digital en Jalisco, México. Comunicación y Sociedad, 30, 126-165. http://doi.org/10.32870/cys.v0i30.683

Bennet, S.; K. Maton (2011). Intellectual Field of Faith-Base Religion. Moving on from the Idea of "Digital Natives". Deconstructing Digital Natives. Young People, Techonology and the New Literacies. [Kindle Edition]. https://www.amazon.com.mx/ 
Burbules, N. (2012). El aprendizaje ubicuo y el futuro de la enseñanza. Encuentros sobre Educación, 13, 3-14. https://doi.org/10.24908/eoe-ese-rse.v13i0.4472

Churches, A. (1/4 de 2008). Bloom's Taxonomy Blooms Digitally. teachLEARNING. Bloom\%27s Taxonomy Blooms Digitally Tech Learning.pdf

Conole, G. (2013). Tools and Resourses to Guide Practice. En Beetham, H.; R. Sharpe (eds.). Rethink Pedagogy for a Digital Age. Nueva York: Routledge, 120-150.

Cope, B.; M. Kalantzis (2009). Ubiquitous Learning. Urbana: University of Illinois Press.

Diario Oficial de la Federación (01 de Enero de 2019). Ley General de Educación. https://www.sep. gob.mx/work/models/sep1/Resource/558c2c24-0b12-4676-ad90-8ab78086b184/ley general educacion.pdf

Gros, B. (2011). El modelo educativo basado en la actividad de aprendizaje. Evolución y retos de la Educación Virtual. Construyendo el e-learning del siglo XXI. Barcelona: UOC, 13-26.

Herrington, J.; T. Reeves; R. Oliver (2010). A Guide to Authentic e-learning. Nueva York: Routledge. Hess, C.; E. Ostrom (2007). Introduction: An Overview of the Knowledge Commons. En Hess, C.; E. Ostrom (eds.). Understanding Knowledge as a Commons. Cambridge: MIT Press, 3-26.

INEGI (2020). Comunicado de Prensa 103/20. https://www.inegi.org.mx/contenidos/saladeprensa/boletines/2020/OtrTemEcon/ENDUTIH 2019.pdf

Koehler, M.; P. Mishra (2009). What is Technological Pedagogical Content Knowledge? Contemporary Issues in Technology and Teacher Education, 9(1), 60-70.

Major, C. (2015). Teaching Online: A Guide to Theory, Research, and Practice. Baltimore: Johns Hopkins University Press.

Martin, A. (2008). Digital Literacy and the "Digital Society". En Lankshear, C.; M. Knobel (eds.). Digital Literacies. Nueva York: Peter Lang, 151-170.

Ostrom, E. (1990). Governing the Commons: The Evolution of Institutions. Nueva York: Cambridge University Press.

Prensky, M. (2001). Digital Natives, Digital Immigrants. On the Horizon, 9(5), 1-6.

Rheingold, H. (2004). Multitudes inteligentes. La próxima revolución social. Barcelona: Gedisa.

Romero-Tena, R.; J. Cabero-Almenara (2007). Bases generales para el diseño, producción y evaluación de las TIC en los procesos de formación. En Cabero-Almena, J.; R. Romero-Tena. Diseño y producción de TIC para la formación. Bacelona: UOC, 29-46.

Rosales-Medrano, M. (2009). La formación profesional del docente de primaria. Cd. Mexico: UPNPlaza y Valdez.

Van Dijk, J. (2017). Digital Divide: Impact of Access. En Rössler, P. (ed.). The International Encyclopedia of Media Effects. Wiley \& Sons, 1-10. http://doi.org/10.1002/9781118783764.wbie$\underline{\text { me0043 }}$ 